INPLASY

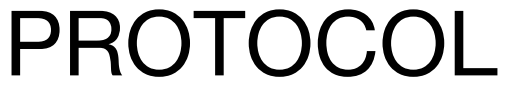

To cite: Tu et al. The efficacy and safety of warming acupuncture and moxibustion combined with Chinese herb medicine on knee osteoarthritis A protocol for a systematic review and meta-analysis. Inplasy protocol 202230012. doi:

10.37766/inplasy2022.3.0012

Received: 03 March 2022

Published: 03 March 2022

Corresponding author: Hongya Yang

36203807@qq.com

Author Affiliation:

Chengdu University of

Traditional Chinese Medicine.

Support: The school funds.

Review Stage at time of this submission: The review has not yet started.

Conflicts of interest:

None declared.

\section{The efficacy and safety of warming acupuncture and moxibustion combined with Chinese herb medicine on knee osteoarthritis A protocol for a systematic review and meta-analysis}

\author{
Tu, S1; Zheng, Y2; Jin, N3; Yang, $\mathrm{H}^{4}$.
}

Review question / Objective: P: Patients with knee osteoarthritis; I: The treatment group includes warming acupuncture and moxibustion combination with Chinese herb medicine at least; $C$ : while the control group is the comparison of western medicine or other physical therapy.; 0 : overall response rate, pain score (such as the visual analogue scale [VAS]); joint function score (such as Lysholm score, Western Ontario and McMaster Universities Arthritis Index [WOMAC], and other scoring systems), daily life quality score (such as SF-36 score [the MOS item short from health survey]), and adverse reactions.; S: Randomized controlled trial.

Condition being studied: Knee osteoarthritis has become a major public health problem, It could cause knee joint pain, joint instability and dysfunction, and seriously affects the quality of life of patients, The purpose of this systematic review is to evaluate the better efficacy and safety of warming acupuncture and moxibustion combined with Chinese herb medicine in the treatment of knee osteoarthritis (KOA) so as to provide comprehensive evidence for the selection of the optimal physical therapy regimens in the clinical treatment ofKOA.

INPLASY registration number: This protocol was registered with the International Platform of Registered Systematic Review and Meta-Analysis Protocols (INPLASY) on 03 March 2022 and was last updated on 03 March 2022 (registration number INPLASY202230012).

\section{INTRODUCTION}

Review question / Objective: P: Patients with knee osteoarthritis; I: The treatment group includes warming acupuncture and moxibustion combination with Chinese herb medicine at least; C: while the control group is the comparison of western medicine or other physical therapy.; 0 : overall response rate, pain score (such as the visual analogue scale [VAS]); joint function score (such as Lysholm score, 
Western Ontario and McMaster Universities Arthritis Index [WOMAC], and other scoring systems), daily life quality score (such as SF-36 score [the MOS item short from health survey]), and adverse reactions.; $S:$ Randomized controlled trial.

Condition being studied: knee osteoarthritis has become a major public health problem, It could cause knee joint pain, joint instability and dysfunction, and seriously affects the quality of life of patients, The purpose of this systematic review is to evaluate the better efficacy and safety of warming acupuncture and moxibustion combined with Chinese herb medicine in the treatment of knee osteoarthritis (KOA) so as to provide comprehensive evidence for the selection of the optimal physical therapy regimens in the clinical treatment ofKOA.

\section{METHODS}

Participant or population: All participants were diagnosed with KOA, participants were not restricted by race and gender.

Intervention: Studies involving, during the course of treatment, the method of warming acupuncture and moxibustion. the kind and taking method of medicine was not restricted, and the time and frequency of warming acupuncture and moxibustion combined with Chinese herb medicine were kept fixed.

Comparator: The control group was treated with western medicine treatment or other physical therapy.

Study designs to be included: The eight databases will be searched from the inception to march 2022. The research on warming acupuncture and moxibustion combined with Chinese herb medicine on knee osteoarthritis meets the screening criteria, and citation screening, data extraction, and risk assessment of bias were performed by two independent reviewers. Cochrane Review Manager 5.3 software was used for statistical analysis.
Eligibility criteria: RCTs evaluating warming acupuncture and moxibustion combined with Chinese herb medicine treatment of KOA will be included. Non-randomized controlled trial, observational research, case reports were excluded. The language of the literature was Chinese or English.

Information sources: The literature search will be identified by eight databases, the Cochrane library, Pubmed, Embase, Web of Science, and four Chinese databases including WANFANG, CNKI, CBM, and VIP database,We searched these databases from their inception at march 2022. Literature language will be excluded except English and Chinese. if necessary, we will contact the author of the original article for more information or clarification. If accurate data cannot be obtained. The available data will be analyzed.

Main outcome(s): The primary efficacy endpoint was the improvement of overall response rate, pain score (such as the visual analogue scale [VAS]); The secondary efficacy endpoint include joint function score (such as Lysholm score, Western Ontario and McMaster Universities Arthritis Index [WOMAC], and other scoring systems), daily life quality score (such as SF-36 score [the MOS item short from health survey]), and adverse reactions. The results of dichotomous data were expressed as a risk ratio with $95 \% \mathrm{Cl}$, for continuous data, we used mean difference or standardized mean difference to evaluate the effect of treatment with $95 \%$ Cl.

Quality assessment / Risk of bias analysis: Two reviewers will assess the risk of bias using the cochrane collaboration tool. The content of the assessment includes: generation of random sequence (selection bias), allocation concealment (selection bias), blinding participants and personnel (performance bias), results blinding of evaluators (detection bias), incomplete result data(attrition bias), selective reporting(reporting bias)and other sources of bias, the risk of bias will be rated as "low risk", "high risk" or "unclear risk" in each area. 
Strategy of data synthesis: The literature search will be identified by eight databases, the cochrane library, pubmed, embase, web of science, and four Chinese databases including WANFANG, CNKI, CBM, and VIP database, we searched these databases from their inception at march 2022. Literature language will be excluded except English and Chinese.

Subgroup analysis: We will conduct subgroup analysis to detect the sources of heterogeneity, including general information (age, sex), type of acupuncture and Moxa cone, course of treatment, type of control, results measurement.

Sensitivity analysis: We conduct sensitivity analysis to screen out high-risk bias and ensure the robustness of the results, based on the missing data, sample size and research characteristics, we will re-analyze and compare the differences with the original data to evaluate the robustness.

Country(ies) involved: China.

Keywords: knee osteoarthritis, warming acupuncture and moxibustion combined with Chinese herb medicine, systematic review.

Contributions of each author:

Author 1 - Shijuan Tu.

Email: 1148217370@qq.com

Author 2 - Yali Zheng.

Author 3 - Ni Jin.

Author 4 - Hongya Yang.

Email: 36203807@qq.com 